\title{
Effect of particle shape on bubble dynamics in bubbling fluidized bed
}

\author{
Siddhartha Shrestha and Zongyan Zhou* \\ ARC Research Hub for Computational Particle Technology, Department of Chemical Engineering, Monash University, Victoria \\ 3800, Australia
}

\begin{abstract}
Particle shape can significantly affect the bubble dynamics of bubbling fluidized beds (BFB). In this paper, findings obtained from simulations using CFD-DEM are summarized to discuss the effect of particle shape on bubble dynamics and bubble properties such as bubble size, shape and velocity at a single orifice and uniform fluidized bed. Particles with aspect ratios at 0.5 (oblate), 1 (spherical) and 2 (prolate) are employed to represent disc-like, spherical and rod-like particles, respectively. Both single jet and uniform fluidized bed simulations demonstrate that the bubble forming/rising regions, bubble coalescence locations, and bubble splitting phenomena are significantly influenced by particle shape. The CFD-DEM results for bubble size and bubble velocity show good agreement with literature correlations.
\end{abstract}

\section{Introduction}

Gas-solid fluidized beds have been widely used in many industrial applications such as food, pharmaceuticals, energy and the environment since they are associated with good heat and mass transfer and gas-solid mixing. The gas-solid fluidized beds have very complex gas and particle flow behaviours due to strong interaction between fluid-particle and particle-particle. In bubbling fluidized beds (BFB), the bubbles govern the gas-solid hydrodynamics, induce gas-solid mixing and promote heat and mass transfer [1]. Thus, bubble dynamics play an essential role to aid in the design and development of gassolid fluidized beds.

The importance of bubble dynamics has led to various experimental investigations such as to portray bubble formation at an orifice [2] and bubble dynamics in uniform fluidized beds [3]. However, experimental studies have limitations that can be easily overcome by numerical simulation. Recently, numerical techniques have been widely used to study gas-solid fluidized beds and have become a significant approach to characterize bubble behaviour. Numerical techniques for gas-solid systems can be either continuum- or discrete-based with respect to the solid phase. The former is called the twofluid model (TFM), where both gas and solid phases are considered continuous and fully interpenetrating. In comparison, the discrete element model (DEM) simulations have an advantage over TFM simulations because the solid phase with respect to friction is modelled much more accurately. In DEM, the particle phase is modelled as a discrete phase and the gas phase as continuous. Several authors have used both TFM and DEM to study bubble dynamics [4-8]. However, most of the previous studies were focused on spherical particles.
In industrial practice, generally, the particles used in BFB are non-spherical. The anisotropic geometry of the nonspherical particles makes the fluidization of non-spherical particles more complex and result in different pressure drop, particle behaviours and mixing behaviour compared to spherical particles $[9,10]$. However, the studies on the effect of particle shape on bubble dynamics in single jet/ uniform bubbling fluidized bed, are not many as expected.

In this paper, we review the key findings obtained from our previous studies that portrayed the effect of particle shape on bubble formation process, bubble dynamics and bubble properties at a single orifice and uniform fluidized bed using CFD-DEM.

\section{Models and simulation conditions}

In CFD-DEM, the gas phase is modelled as TFM and the solid phase is based on the DEM. The gas phase is modelled as a continuum and the volume-averaged Navier-Stokes equations are solved to obtain the fluid dynamics. While the solid phase is modelled as discrete particles and the motion of individual particles is tracked in each time-step by solving Newton's second law of motion. The coupled CFD-DEM technique has been widely used to perform fluidized bed simulations $[8,9$, $11,12]$. The details of the governing equations and the equations to calculate the interaction forces and torques are well documented and interested readers can refer to our previous works [12-14].

The bed geometry is a slot model with periodic boundary conditions applied to the front and rear direction. For such a geometry, two-dimensional CFD and three-dimensional DEM are employed. Initially, a static bed is generated by using the gravitational settling process. Then, two gas inlet conditions are simulated (i)

\footnotetext{
* Corresponding author: zongyan.zhou@monash.edu

A video is available at https://doi.org/10.48448/v89p-gk04
} 
single jet fluidized bed and (ii) uniform fluidized bed. For a single jet fluidized bed, the bed is fluidized by injecting a gas jet at the central orifice while the lateral region is maintained at minimum fluidization velocity $\left(U_{m f}\right)$. For a uniform fluidized bed, the bed is fluidized at 4 and 6 times of $U_{m f}$.

The bubble properties were identified by defining the bubble boundary with a threshold void fraction value of 0.8. The geometric properties of bubbles were determined in MATLAB by using the Image Processing Toolbox. Details regarding the procedure to obtain bubble properties are available in our previous studies [13, 14].

\section{Key findings}

\subsection{Single jet fluidized bed}

When a continuous jet of air is injected from the central orifice to the static bed of particles, a series of bubbles are formed one after another at a certain distance above the distributor. Particle shape can significantly affect the bubble formation process (bubble splitting and coalescence), and bubble size, shape and velocity. The time for bubble formation and the time for detachment is slightly sensitive to particle shape (Fig. 1). In comparison, the bubble splitting and bubble coalescence process are more sensitive. The bubbles generally split at the lower level of the bed which merges for spheres but for ellipsoids they behave as different bubbles while rising. The location of bubble coalescence for particles of different shapes are similar and usually at three different locations (low, mid and top level of the bed) as shown in Fig. 2, however, the patterns of bubble coalescence are different and depend upon particle shape [13].
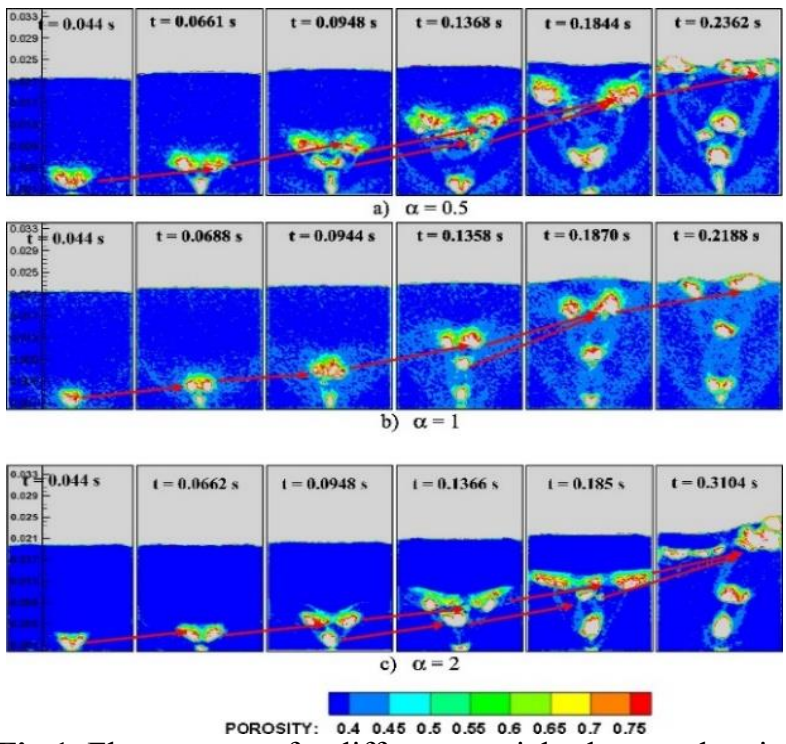

Fig.1. Flow patterns for different particle shapes when jet velocity is $0.1 \mathrm{~m} / \mathrm{s}$ (colored by porosity): (a) oblate particles, (b) spheres, and (c) prolate particles [13].

Figs. 3, 4 and 5 show the averaged bubble diameter, bubble shape factor and bubble velocity evaluated for each particle shape. Both bubble diameter and velocity increases while the bubble shape factor decreases with the increase of the jet velocity. While for a specific jet velocity, the bubble diameter is minimum for spheres compared to ellipsoids. This is due to the low minimum fluidization velocity of ellipsoids compared to spheres. Lower minimum fluidization velocity gives rise to larger bubble size and less gas leakage. In contrast, the bubble shape factor and the bubble velocity for spheres are highest compared to ellipsoids for all jet velocities. The bubble rising velocity for ellipsoids is lower because the bubbles need to break the interlocks between the particles and allow the particles to rotate to their preferred orientation $[13,15]$.
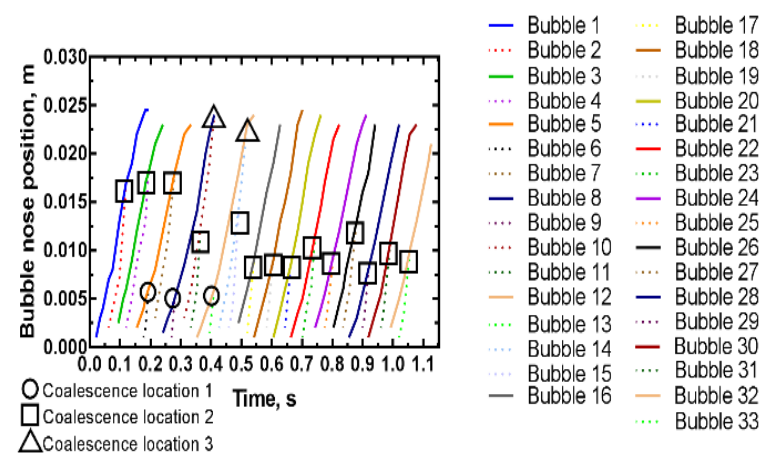

Fig. 2. The motion of a chain of bubbles for a jet velocity of $0.15 \mathrm{~m} / \mathrm{s}$ (straight lines indicate primary bubbles and dotted lines indicate trailing bubbles) [16].

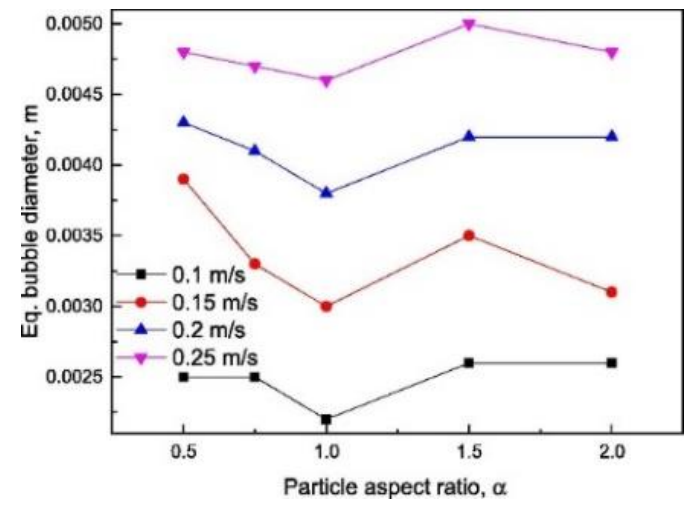

Fig. 3. Equivalent bubble diameter as a function of particle shape at different jet velocities [13].

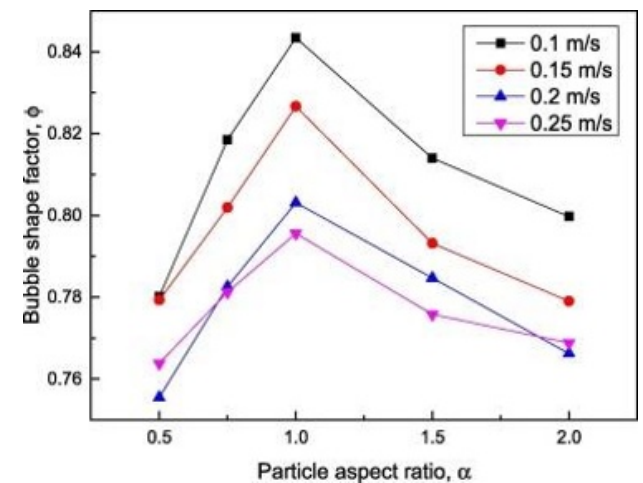

Fig. 4. Bubble shape factors for different particle shapes and jet velocities [13].

Besides, jet velocity, the cohesive force expressed in terms of van der Waals force is another factor that can 
significantly enhance the particle shape effect. With increasing cohesive force, the time of bubble detachment increases, the instances of bubble splitting and the number of locations of bubble coalescence decreases (Fig. 6 - for brevity flow patterns for other particles are not shown and can be referred [17]). Moreover, both bubble diameter and bubble velocity decrease with the increase of cohesive force (Figs. 7 and 8).

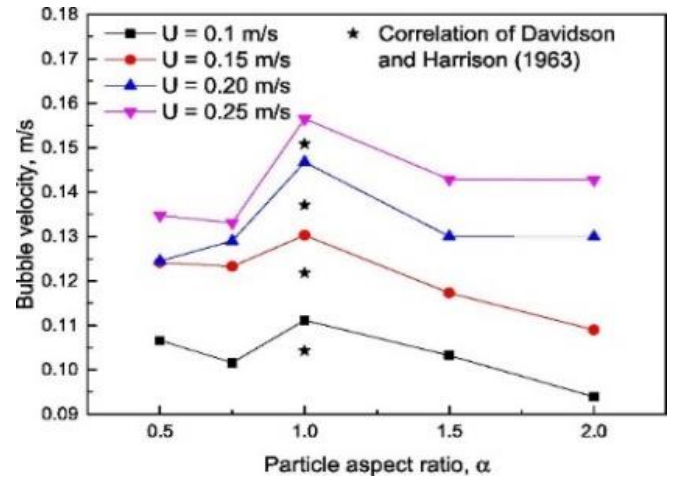

Fig. 5. Bubble velocity as a function of particle shape and jet velocity [13].

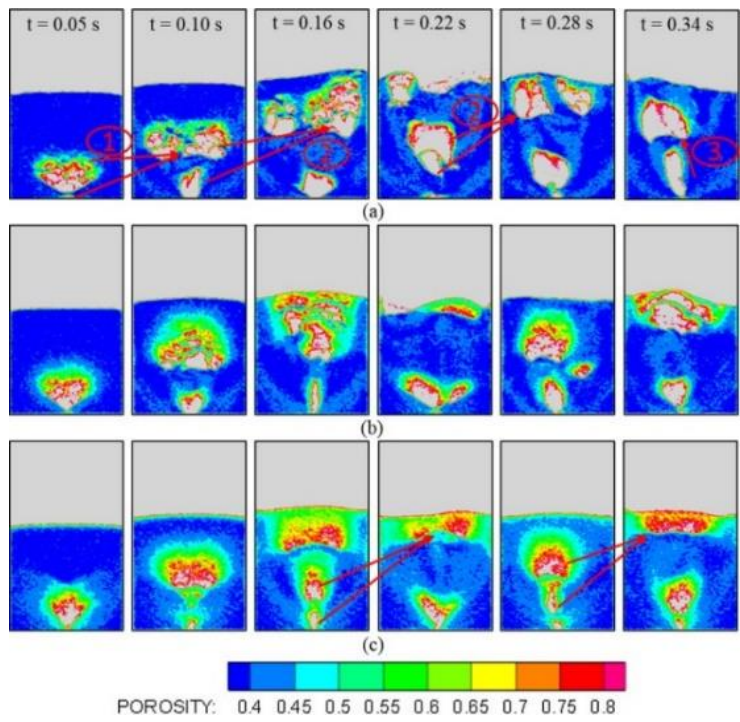

Fig. 6. Flow patterns for oblate ellipsoids $(\alpha=0.5)$ at jet velocity of $0.3 \mathrm{~m} / \mathrm{s}$ with different Hamaker constant: (a) $0 \mathrm{Ha}$, (b) $2 \mathrm{Ha}$, and (c) $15 \mathrm{Ha}$, colored by porosity [17].

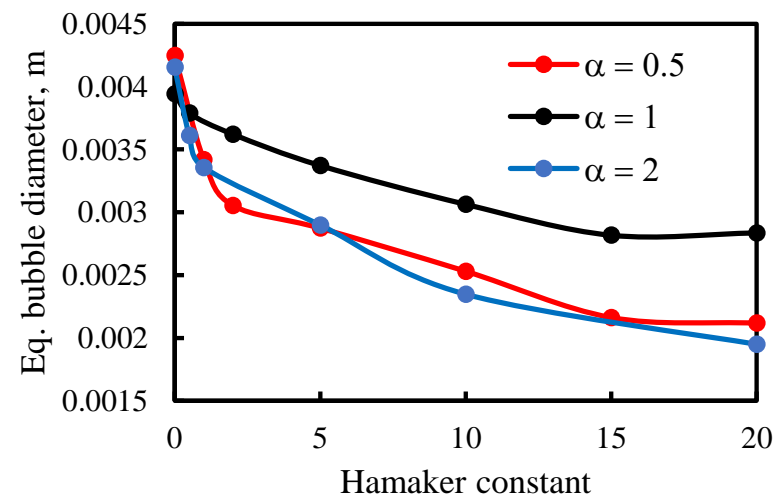

Fig. 7. Equivalent bubble diameter at different Hamaker constant for different particle shapes at jet velocities of 0.20 $\mathrm{m} / \mathrm{s}$ [17].

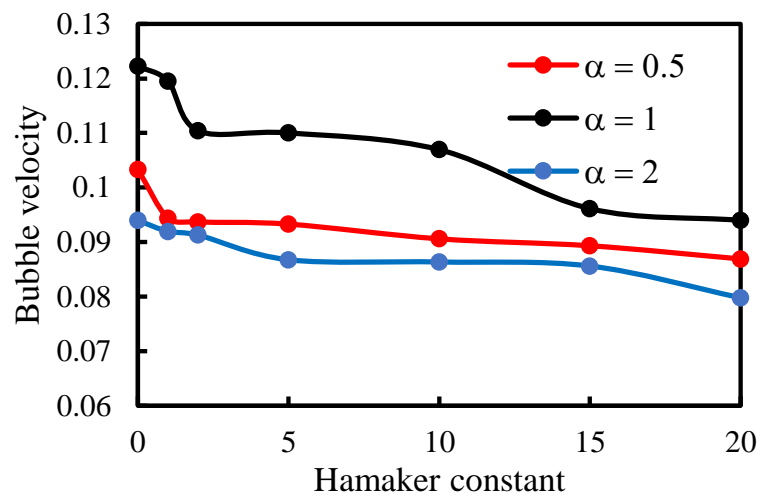

Fig. 8. Bubble rising velocity at different Hamaker constant when jet velocity is $0.30 \mathrm{~m} / \mathrm{s}$ [17].

\subsection{Uniform fluidized bed}

When a gas flow is uniformly injected into a packed bed of particles, the bed is fluidized, and the bubble are generated throughout the bed (Fig. 9). During the bubble rising process, the bubbles can coalesce to form bigger bubbles or split to smaller ones. In the figure, the black and red circles show samples of different coalescence mechanisms. For spheres $(\alpha=1)$, the bed materials move upward from the center of the bed while they descend downward in the annulus. On the contrary, ellipsoids have asymmetrical down-flow patterns and are not ideally located at the annulus in the bed. The difference in particle shape also leads to changes in gas velocity distribution, particle velocity distribution, up-flow and down-flow mass flux, and mixing properties [14]. This difference becomes more noticeable at higher velocity.

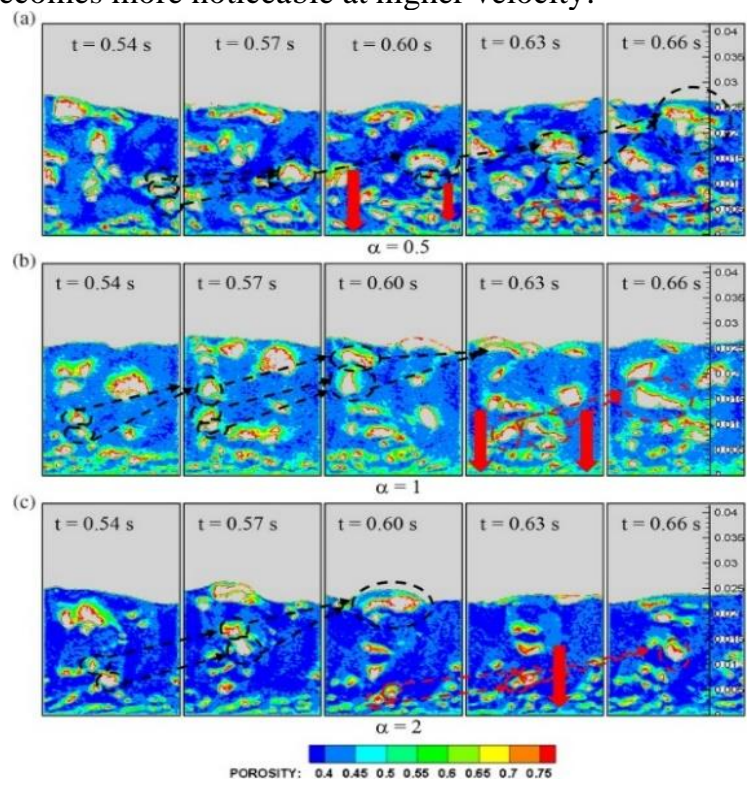

Fig. 9. Typical images of fluidized bed at $4 U_{m f}$ for different particle shapes. (a) $\alpha=0.5$, (b) $\alpha=1$, and (c) $\alpha=2$ [14].

The bubble diameter and bubble velocity increase with the increase of the gas velocity (Figs. 10 and 11). The spheres have the highest bubble diameter and the highest bubble velocity. Moreover, the change in bubble diameter along with the bed height and the change in bubble velocity with gas velocity calculated from CFD-DEM 
matches the literature correlations (Figures can be referred in [14]). Both bubble diameter and bubble velocity for a particle of different shapes show dependence on $U_{m f}$. It should be noted that the $U_{m f}$ for different particle shapes is based upon calculation from CFD-DEM, therefore a proper correlation for minimum fluidization velocity should be used for the particles [11].

The fluidization of ellipsoids gradually transforms into a non-bubbling fluidization regime with the increase of the van der Waals force (Fig. 12). However, for spheres, a higher cohesive force can lead to the formation of gas flow channels, showing the behaviour of Geldart C powders. Moreover, the equivalent bubble diameter decreases with the increase of cohesive force for different particle shapes [17].

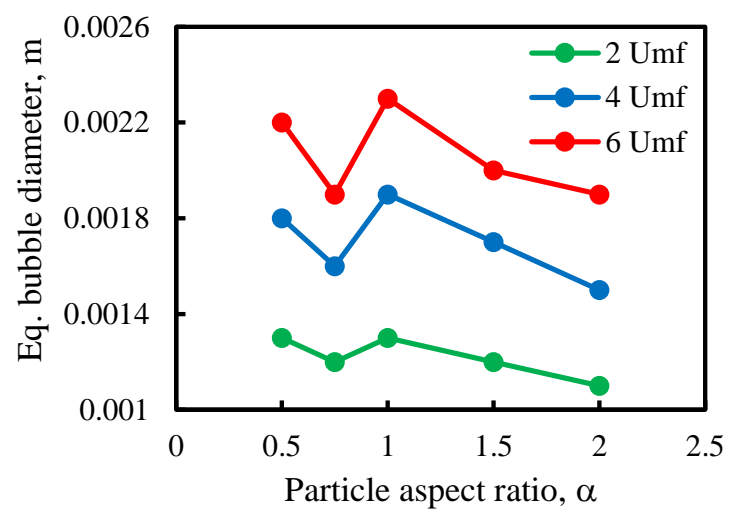

Fig. 10. Equivalent bubble diameter for different particle shape [14].

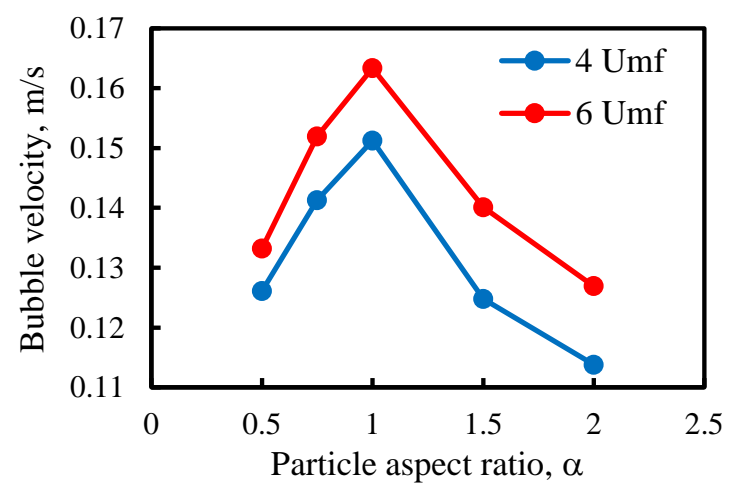

Fig. 11. Bubble velocity for different particle shape [14].

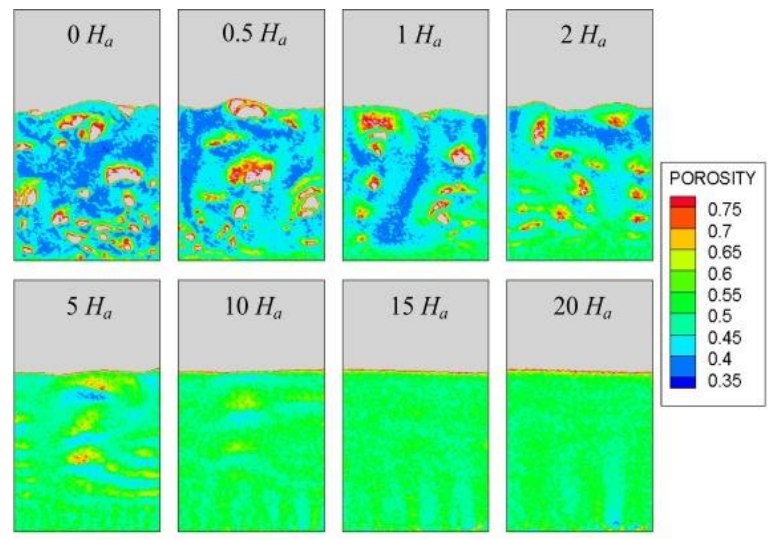

Fig. 12. Flow patterns for oblate ellipsoids $(\alpha=0.5)$ with different Hamaker constant at a gas velocity of $6 U_{m f}[17]$.

\section{Conclusion}

The effect of particle shape on bubble dynamics in a single jet and uniform fluidized bed were investigated using CFD-DEM simulations. To discuss the effect of particle shape, the results were analysed and discussed in terms of bubble rising process and bubble properties for oblate, spheres and prolate particles. The simulation results demonstrated that the bubble flow and particle flow behaviour are dependent upon particle shape. Moreover, the obtained results from CFD-DEM for bubble diameter, shape and velocity showed a significant difference for oblate and prolate particles compared to spheres. The conclusions derived from this work are expected to improve the understanding of bubble dynamics in bubbling fluidization of non-spherical particles.

\section{References}

[1] A. Busciglio, G. Vella, G. Micale, L. Rizzuti, Chem. Eng. J. 140, 398-413 (2008)

[2] A.D. Lotfabad, S. Movahedirad, M. Sadeghi, Iran. J. Chem. Eng. 13, 61 (2016)

[3] J.A. Laverman, I. Roghair, M.v.S. Annaland, H. Kuipers, Can. J. Chem. Eng. 86, 523-535 (2008)

[4] Y. He, S. Yan, T. Wang, B. Jiang, Y. Huang, Powder Technol. 287, 264-276 (2016)

[5] R.P. Utikar, V.V. Ranade, Chem. Eng. Sci. 62, $167-$ 183 (2007)

[6] O.O. Olaofe, M.A. van der Hoef, J.A.M. Kuipers, Chem. Eng. Sci. 66, 2764-2773 (2011)

[7] L.-W. Rong, J.-M. Zhan, C.-L. Wu, Adv. Powder Technol. 23,398-405 (2012)

[8] Y. Lu, J. Huang, P. Zheng, Chem. Eng. J. 274, 123 $131(2015)$

[9] H. Ma, L. Xu, Y. Zhao, Powder Technol. 314, 355366 (2017)

[10] K. Vollmari, R. Jasevičius, H. Kruggel-Emden, Powder Technol. 291, 506-521 (2016)

[11] J. Gan, Z. Zhou, A. Yu, AIChE Journal 62, 62-77 (2016)

[12] Z. Zhou, D. Pinson, R. Zou, A. Yu, Chem. Eng. Sci. 66, 6128-6145 (2011)

[13] S. Shrestha, S. Kuang, A. Yu, Z. Zhou, Chem. Eng. Sci. 201, 448-466 (2019)

[14] S. Shrestha, S. Kuang, A. Yu, Z. Zhou, AIChE Journal 65, e16736 (2019)

[15] S. Shrestha, S. Kuang, A. Yu, Z. Zhou, Powder Technol. 361, 363-373 (2020)

[16] S. Shrestha, J. Gan, Z. Zhou, Powder Technol. 357, 398-407 (2019)

[17] S. Shrestha, S.B. Kuang, A.B. Yu, Z.Y. Zhou, Chem. Eng. Sci. 212, 115343 (2020) 